\title{
Experimental method based on wavelength-modulation spectroscopy for the characterization of semiconductor lasers under direct modulation
}

\author{
Stéphane Schilt and Luc Thévenaz
}

\begin{abstract}
An experimental method is presented for characterization of the combined intensity and frequency modulation produced when the injection current of a laser diode is modulated. The reported technique is based on the analysis of the harmonic signals produced when a modulated laser is used to probe a gas absorption line by the so-called wavelength-modulation spectroscopy method. Based on a theoretical model of this technique, we present two methods that facilitate the determination of (i) the deviation in laser frequency and (ii) the phase shift between intensity and frequency modulation. These methods are illustrated experimentally by measurement of the modulation parameters of a 2 - $\mu \mathrm{m}$ distributed-feedback laser by use of $\mathrm{a} \mathrm{CO}_{2}$ absorption line. The experimental results have been compared with those obtained with another traditional method and have shown full agreement in the frequency range $(400 \mathrm{~Hz}-30 \mathrm{kHz})$ considered. () 2004 Optical Society of America
\end{abstract}

OCIS codes: $140.5960,300.6380,120.3940,300.6260$.

\section{Introduction}

Wavelength-modulation spectroscopy (WMS) is a widely used technique for trace-gas detection by use of laser diodes in the near and mid infrared.1-16 This sensitive technique is based on sinusoidal modulation of the light emitted by a laser diode that is slowly tuned through an absorption feature of the species to be detected. The interaction of the modulated light with the absorption feature leads to the generation of signals at the different harmonics of the modulation frequency, which can be detected with a lock-in amplifier. These harmonic signals are directly proportional to the absorption and are usually used to monitor gas concentration. Another original application of this technique is presented here. It consists in the experimental measurement of the modulation parameters of a laser diode by use of the harmonic signals produced when an absorption line

The authors are with Ecole Polytechnique Fédérale de Lausanne, Swiss Federal Institute of Technology, Laboratory of Nanophotonics and Metrology, CH-1015 Lausanne, Switzerland. S. Schilt's e-mail address is stephane.schilt@epfl.ch.

Received 2 September 2003; revised manuscript received 22 March 2004; accepted 21 May 2004.

0003-6935/04/224446-08 $\$ 15.00 / 0$

(C) 2004 Optical Society of America of known parameters is probed by the modulated laser.

Laser diodes are easily and efficiently modulated by dithering of their injection current. This results in simultaneous intensity modulation (IM) and frequency modulation (FM) of the emitted light. Furthermore, a phase shift is observed between the two modulations; the phase varies from 0 at low modulation frequencies to $-\pi$ at high frequencies. ${ }^{17}$ Various experimental techniques, usually based on the use of a high- or a low-finesse interferometer, have been proposed for measuring the modulation parameters of a laser diode. ${ }^{18-21}$ One of these methods, which facilitates the measurement of both the deviation in laser frequency and the IM-FM phase shift, is briefly reviewed in Section 2 of this paper. Then our measurement method, derived from our work on trace-gas detection by WMS, is presented. WMS is based mainly on FM of laser light, whereas IM is an undesirable effect that distorts the signals. In a previous paper, Schilt et al. presented a general analytical model describing the harmonic signals obtained when a Lorentzian absorption line is probed by a modulated laser. ${ }^{22}$ This model takes into account the combined IM-FM of a laser diode with an arbitrary phase shift and shows the effect of several modulation parameters on the detected signals. Here we briefly review the main results of this model to apply them to the determination of the modulation 
parameters of a laser diode. Different methods are proposed for the measurement of the deviation in laser frequency and of the IM-FM phase shift. These methods are illustrated by experimental results obtained with a $2-\mu \mathrm{m}$ distributed-feedback (DFB) laser by use of a $\mathrm{CO}_{2}$ absorption line at atmospheric pressure. These results are compared with those of another traditional experimental technique and the performances are discussed.

\section{IM-FM in a Laser Diode: Description and Measurement Techniques}

The modulation of injection current $i$ in a laser diode produces simultaneous modulation of optical intensity $I$ and of laser instantaneous frequency $v$ :

$$
\begin{array}{ll}
I(t)=I_{0}(1+M \cos \omega t) & (I M), \\
v(t)=v_{0}-\Delta v \cos (\omega t+\Psi) & (F M),
\end{array}
$$

where $M$ is the intensity modulation index, $\omega$ is the modulation angular frequency, and $\Psi$ is the phase shift between IM and FM. A negative sign is chosen in the expression for FM to produce zero phase shift $\Psi$ at low frequency in the common case of DFB lasers (the laser frequency decreases in a DFB laser when the optical power increases). Various experimental methods to measure the modulation parameters of a laser diode have been proposed. For example, one can use a Fabry-Perot analyzer to determine the FM efficiency through direct observation of the modulation spectrum. ${ }^{18,23}$ However, this technique requires expensive mirrors with high reflectivity and does not permit precise measurement of the IM-FM phase shift. Another, more attractive, technique facilitates the simultaneous determination of the FM efficiency and the IM-FM phase shift. It is based on an unbalanced interferometric method proposed by Olesen and Jacobsen ${ }^{21}$ for measurement of the phase shift.

This method was initially presented in a Michelson configuration. To validate the novel measurement technique presented in this paper we implemented the interferometric method, using a Mach-Zehnder configuration. This modification has enabled us to reduce the optical feedback into the laser and to improve the stability of the signals, particularly when it is used with nonisolated lasers. The two arms of the interferometer have different lengths, $L_{1}$ and $L_{2}$, as illustrated in Fig. 1. Output signal $I_{\text {out }}(t)$ of the interferometer is given by ${ }^{21-23}$

$$
\begin{aligned}
I_{\text {out }}(t)= & (1+M \cos \omega t)\left\{1+R_{0}(\Delta T) \cos \right. \\
& \left.\times\left[2 \pi \Delta \nu \Delta T \cos \left(\omega t^{\prime}+\Psi\right)-2 \pi v_{0} \Delta T\right]\right\},
\end{aligned}
$$

where $t^{\prime}=t-T_{0}$ and $R_{0}(\Delta T)$ is the autocorrelation function of the electrical field. In Eq. (2), the average propagation time $T_{0}$ and the difference $\Delta T$ in the propagation times in the two arms of the interferometer are given by $T_{0}=\left(T_{1}+T_{2}\right) / 2$ and $\Delta T=\left(T_{1}-\right.$ $T_{2}$ ), where $T_{1}$ and $T_{2}$ represent the propagation times

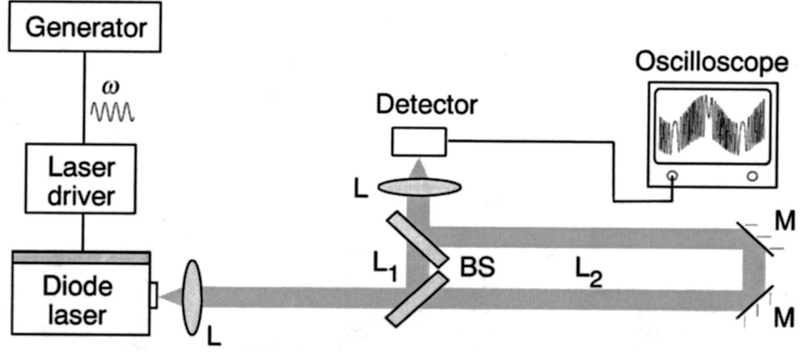

Fig. 1. Experimental schematic of the unbalanced interferometer used for measurement of IM-FM phase shift $\Psi$ : M's, mirrors; BS, beam splitter; L's, lenses.

in the two arms of the interferometer. Equation (2) is valid when the condition

$$
\frac{\omega \Delta T}{2} \ll 1
$$

is fulfilled. The detected signal consists in an interference pattern superimposed upon a sinusoidal envelope produced by the laser's IM. It contains a term, $\cos \left[2 \pi A \cos \left(\omega t^{\prime}+\Psi\right)\right]$, that shows some symmetry axes (see Fig. 2) located in

$$
t_{k}=\frac{-\Psi}{2 f} \pm \frac{k}{2 f}, \quad k=0,1,2,3, \ldots
$$

The IM-FM phase shift may be determined by delay $t_{k}$ between one of the symmetry axes (points where the frequency of the cosine term equals zero) and the IM maximum. The IM-FM phase shift is thus directly extracted from the interference signal, as shown in Fig. 2. This unbalanced interferometric method can also be used for the measurement of the FM efficiency. The number of interference periods between two successive symmetry axes in the detected signal is directly proportional to the laser frequency deviation $\Delta v$ :

$$
N_{\text {fringes }}=2 \Delta v \Delta T=2 \Delta v \frac{\Delta L}{c},
$$

where $c$ is the velocity of light. When length difference $\Delta L$ between the two arms of the interferometer is known, the FM amplitude is directly determined from the number of fringes:

$$
\Delta v=\frac{N_{\text {fringes }} c}{2 \Delta L} .
$$

\section{Application of WMS to the Characterization of a Modulated Laser}

The theoretical model of WMS that we have developed applies to a Lorentzian absorption line scanned by a laser diode that is simultaneously modulated in intensity and in frequency, with an arbitrary phase shift $\Psi .^{22}$ Laser modulation frequency $f$ is generally bound in a range of some hundreds of hertz to some hundreds of kilohertz, whereas a scan is performed through the absorption line by use of a slow current 
(a)

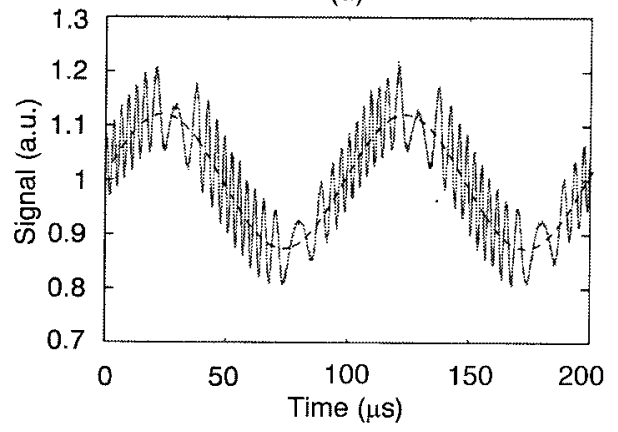

(b)

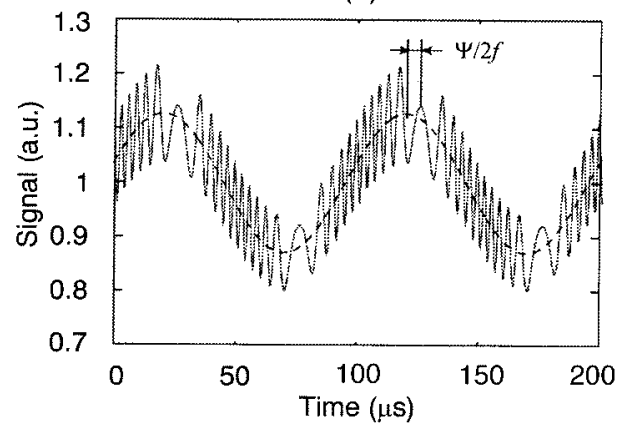

Fig. 2. Output signal from the unbalanced interferometer (solid curves). The dashed curves represent the signal without the interference fringes. Phase shift $\Psi$ is measured between the maximum of this curve and the symmetry axis of the signal. (a) Experimental signal obtained with a DFB laser emitting at $\lambda=2 \mu \mathrm{m}$, with $L_{2}-L_{1}=76 \mathrm{~cm}, f=10 \mathrm{kHz}, M=0.128, \Delta v=2.1 \mathrm{GHz}, \Psi=-22 \mathrm{deg} . \quad(\mathrm{b})$ Simulated signal given by Eq. (2) for the same parameters.

ramp at a frequency $F$ of a few hertz. This description applies well to gas measurements near atmospheric pressure, where the Voigt line shape is strongly dominated by the Lorentzian contribution and is therefore well approximated by a Lorentzian function. The FM of the laser is described by the instantaneous frequency given in Eq. (1), and the Lorentzian absorption line is given by the line-shape function

$$
g(x)=\frac{1}{\pi \Delta v_{\text {line }}} \frac{1}{1+x^{2}}
$$

where

$$
x=\left(v-v_{\text {line }}\right) / \Delta v_{\text {line }}
$$

is a frequency that has been normalized to the line parameters, $\nu_{\text {line }}$ is the absorption line center, and $\Delta v_{\text {line }}$ is the linewidth (half-width at half-maximum; HWHM). The FM amplitude is characterized by modulation index $m=\Delta v / \Delta v_{\text {line }}$, which represents the maximum deviation in laser frequency normalized by the absorption linewidth. The laser's IM is taken into account by introduction of a linear variation with slope $p$ in the incident optical power as a function of the frequency. As the FM efficiency depends on the modulation frequency, ${ }^{17}$ this coefficient takes a different value for either the sine modulation at the angular frequency $\omega=2 \pi f$ or the lowfrequency ramp used to scan the laser line through the absorption feature. Two different coefficients, $p_{\omega}$ and $p_{\Omega}$, are therefore introduced to describe the variation in laser optical power. ${ }^{22}$ The first one is associated with modulation frequency $\omega$; the second, with the low-frequency ramp at $\Omega=2 \pi F$. The general analytical expressions for the signals detected at the three first harmonics of the modulation frequency were given in detail in a previous paper. ${ }^{22}$ Here we summarize only the main results necessary for the description of the experimental method that we report for the measurement of the modulation parameters.

\section{A. IM-FM Phase Shift Measurement}

The amplitude of the WMS signal detected at the $n$th harmonic varies periodically with phase $\Phi_{n}$ of the lock-in detection. For each harmonic, the amplitude reaches maximum and minimum values at detection phases $\Phi_{n, \max }$ and $\Phi_{n, \min }$, respectively, that are directly related to the IM-FM phase shift ${ }^{22}$ :

$$
\begin{aligned}
& \Phi_{n, \text { max }}=n \Psi+k \pi, \\
& \Phi_{n, \text { min }}=n \Psi+(2 k+1) \pi / 2 .
\end{aligned}
$$

One can exploit this property experimentally to determine the IM-FM phase shift. The method of measurement consists in determining detection phase $\Phi_{n, \min }$ that minimizes the amplitude of the $n$ th-harmonic signal. The phase shift is determined by inversion of Eq. (9b):

$$
\Psi=\frac{\Phi_{n, \min }-(2 k+1) \pi / 2}{n} .
$$

In practice, however, detection phase $\Phi_{n}$ is a relative parameter that is not known precisely, as it depends on some phase shifts in the electronics. It is therefore necessary to determine the minimal amplitude of the signal at two different harmonics, $n_{1}$ and $n_{2}$, and the phase shift is obtained from the difference in the position of these minima:

$$
\Psi=\frac{\Phi_{n_{2}, \min }-\Phi_{n_{2} 1, \min }}{n_{2}-n_{1}} .
$$

In practice, harmonics $n_{1}=2$ and $n_{2}=3$ are commonly used for the determination of the IM-FM phase shift but higher harmonics can be used as well.

\section{B. Measurement of the FM Amplitude}

The FM efficiency in a laser diode is characterized by the parameter $\Delta v / \Delta i[\mathrm{GHz} / \mathrm{mA}]$, where $\Delta i$ is the amplitude of the current modulation. This efficiency can be determined experimentally from the variation of the maximum $\left(s_{n, \max }\right)$ or the amplitude $\left(s_{n, \text { ampl }}\right)$ of the $n$ th-harmonic signal as a function of 
(a)
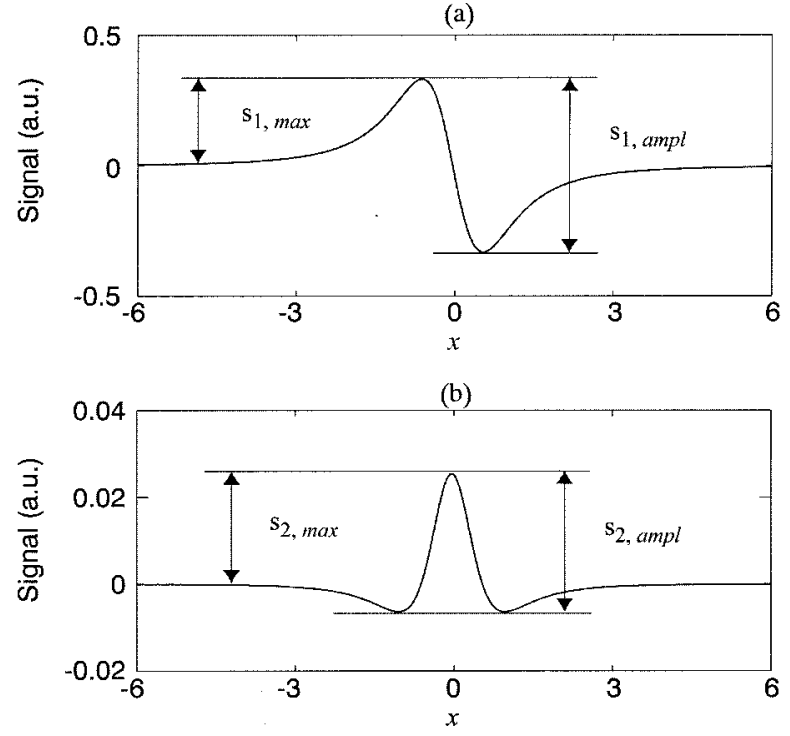

(c)

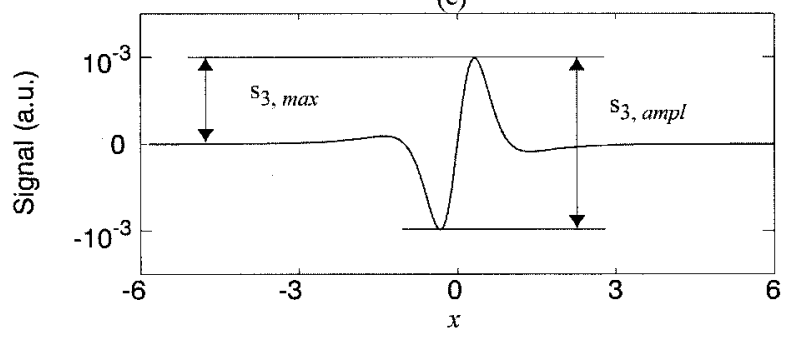

Fig. 3. Shape of the WMS signals obtained at (a) the first, (b) the second, and (c) the third harmonic. Maximum $s_{n, \text { max }}$ and amplitude $s_{n, \text { ampl }}$ of the signal are defined in each case.

modulation amplitude $\Delta i$ applied to the laser. These parameters are defined in Fig. 3 for the first three harmonics. They depend on modulation in$\operatorname{dex} m$, and, for each harmonic, there exist two optimal modulation indices, $m_{n, \max }{ }^{\text {opt }}$ and $m_{n, \text { ampl }}{ }^{\text {opt }}$, for which either $s_{n \text {,max }}(m)$ or $s_{n \text {,ampl }}(m)$ is maximal. Whereas these values are perfectly determined in the case of a pure FM $\left(m_{n, \max }{ }^{\text {opt }}\right.$ is $2.00,2.20,3.59$, and 4.12 for the four first harmonics ${ }^{22,24}$ ), they depend on all the modulation parameters $\left(m, p_{\omega}, p_{\Omega}\right.$, and $\Psi$ ) when a combined IM-FM is considered. However, it was shown that, for realistic values of IM parameters, the optimal values $m_{n, \max }{ }^{\text {opt }}$ for which $s_{n \text {,max }}(m)$ reaches its maximum are not modified from those of pure FM for even harmonics. ${ }^{22}$ On the contrary, these values strongly vary for odd harmonics owing to the asymmetry produced by IM. In this case, the index $m_{n \text {,ampl }}{ }^{\text {opt }}$ that maximizes the total amplitude of the signals is, however, constant when IM increases. The measurement method that we have developed is based on this property and uses the second-harmonic signal. This signal is proportional to the expression

$$
s_{2, \Phi}(x)=s_{2 p}(x) \cos \Phi_{2}+s_{2 q}(x) \sin \Phi_{2},
$$

where $\Phi_{2}$ represents the phase of the lock-in detection and $s_{2 p}(x)$ and $s_{2 q}(x)$ are the $2 f$ signals in phase and in quadrature, respectively:

$$
\begin{aligned}
s_{2 p}(x)= & I_{\Omega}(x) \cos 2 \Psi s_{2}(x)-p_{\omega} \Delta \nu_{\text {line }}(m / 2) \\
& \times\left[\cos \Psi s_{1}(x)+\cos 3 \Psi s_{3}(x)\right], \\
s_{2 q}(x)= & I_{\Omega}(x) \sin 2 \Psi s_{2}(x)-p_{\omega} \Delta \nu_{\text {line }}(m / 2) \\
& \times\left[\sin \Psi s_{1}(x)+\sin 3 \Psi s_{3}(x)\right] .
\end{aligned}
$$

In Eqs. (13), $I_{\Omega}(x)=p_{\Omega} \Delta v_{\text {line }} x+1$, whereas the functions $s_{n}(x)$ correspond to the signal at the $n$th harmonic obtained for pure FM.1,25 The maximum of the $2 f$ signal is obtained for detection phase $\Phi_{2, \max }=$ $2 \Psi$ according to Eq. (9a). For pure FM the maximum is reached exactly on the line center $(x=0)$. In the general case of combined IM-FM, the maximum is moved slightly from line center. The corresponding deviation $\Delta x_{2}$ depends on modulation parameters $p_{\omega}$ and $p_{\Omega}$. However, we have observed that this shift was weak (lower than $10 \%$ of the absorption linewidth) and mainly that the difference between the maximum of the $2 f$ signal and its value at line center was negligible. This difference was less than 1-2\%, even for modulation parameters two times larger than in real conditions [we measured typical values of $p_{\Omega}=-1\left(1 / \mathrm{cm}^{-1}\right)$ and $p_{\omega}=-2(1 /$ $\mathrm{cm}^{-1}$ ) at $f=10 \mathrm{kHz}$ on a DFB laser emitting at $\left.2 \mu \mathrm{m}\right]$. Consequently the shift of the maximum of the $2 f$ signal from the line center can be neglected for realistic values of the modulation parameters, and one obtains the maximum of the $2 f$ signal by introducing $x=0$ and $\Phi_{2}=2 \Psi$ in into Eq. (13):

$$
s_{2, \max }=I_{0} a_{0}\left(\frac{-4}{m^{2}}+\frac{2}{m^{2}} \frac{m^{2}+2}{\sqrt{m^{2}+1}}\right),
$$

where $I_{0}$ is the optical power at line center and $a_{0}$ is the absorbance at the same point. The function $s_{2, \max }(m)$ is independent of IM, as described above; it reaches its maximum for $m_{2, \text { max }}{ }^{\text {opt }}=\sqrt{2+2 \sqrt{2}} \cong 2.20$. The method of determining the FM index consists in measuring the maximum $s_{2 \text {,max }}$ of the $2 f$ signal as a function of current modulation amplitude $\Delta i$. A fit of the experimental points by function (14) determines a proportionality coefficient $K$ between modulation index $m$ and modulation amplitude $\Delta i$ :

$$
m=\frac{\Delta v}{\Delta v_{\text {line }}}=K \Delta i .
$$

Finally, the FM depth may be determined if absorption linewidth $\Delta v_{\text {line }}$ is known:

$$
\Delta v / \Delta i=K \Delta \nu_{\text {line }}
$$

\section{Experimental Application of the Method}

The FM efficiency and the IM-FM phase shift of a DFB laser diode emitting near a $2-\mu \mathrm{m}$ wavelength have been characterized by the methods presented in Section 3. An absorption line of $\mathrm{CO}_{2}$ at $2004 \mathrm{~nm}$ (line $R 16$ ) was used at atmospheric pressure for de- 


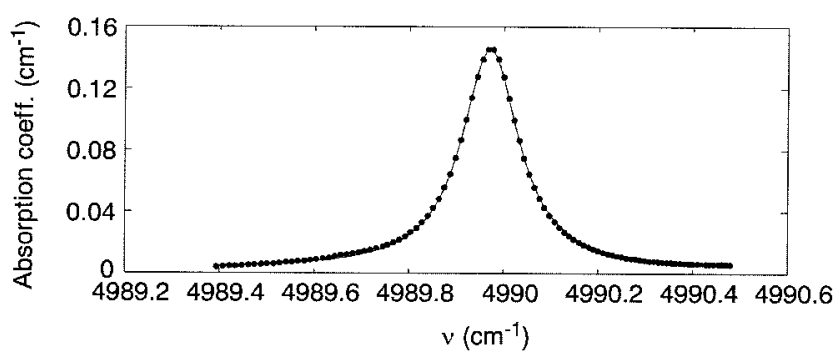

Fig. 4. Absorption line $R 16$ of $\mathrm{CO}_{2}$ measured with a DFB laser emitting at $2004 \mathrm{~nm}$. The laser frequency was measured with a wavemeter with a resolution of $1 \mathrm{pm}$. Experimental points are represented by circles, and the curve results from a fit with a Lorentzian distribution.

termination of the modulation parameters. A scan through this line, which we obtained by varying the laser temperature, is shown in Fig. 4. A Lorentzian fit through the experimental points is also represented and confirms that the line shape is well approximated by a Lorentzian distribution. The line parameters extracted from the fit fully agree with the values given by the HITRAN database, ${ }^{26}$ as listed in Table 1. The theoretical model that we have developed and the experimental methods that we propose with which to determine the modulation parameters of the laser apply therefore properly to gas absorption lines near atmospheric pressure.

The setup used for the experimental verification of our method is shown in Fig. 5. The laser is temperature stabilized and driven by a commercial laser current supply. It is sinusoidally modulated at variable frequency $f$, and a current ramp is applied at $F=$ $\Omega / 2 \pi=9.5 \mathrm{~Hz}$ to scan the absorption line. The laser beam is collimated with an off-axis parabolic mirror and is divided into two paths by a beam splitter. In the first arm, the light beam goes through a measurement cell of length $L=100 \mathrm{~cm}$ and is focused onto an InGaAs photodetector. The second part of the beam is focused onto a reference detector. Signals from both detectors are amplified and subtracted, such that they exactly counterbalance when no absorbing species is present in the cell. The lengths of the two paths outside the cell are equalized, so the absorption that is due to ambient $\mathrm{CO}_{2}$ is suppressed.

The WMS signals obtained at the harmonics $n=2$ and $n=3$ when the cell is filled with ambient air (350 parts in $10^{6}$ of $\mathrm{CO}_{2}$ ) are recorded by an oscilloscope. The background signals produced when the cell is purged with pure $\mathrm{N}_{2}$ are also recorded and are subtracted from the signals obtained with $\mathrm{CO}_{2}$. The

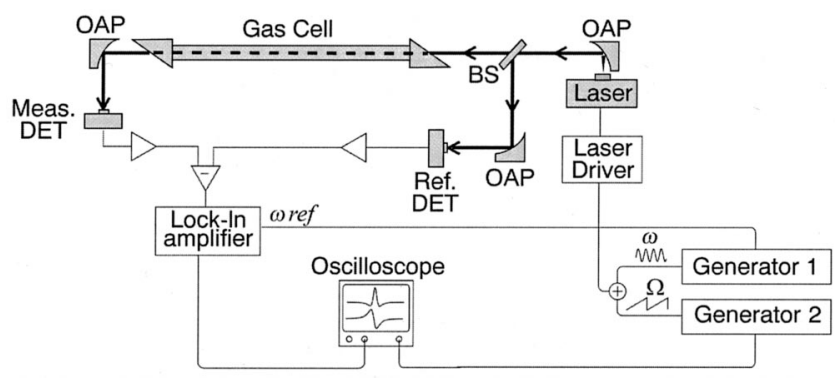

Fig. 5. Experimental setup of the WMS technique: OAPs, offaxis parabolic mirrors; BS, beam splitter; DETs, detectors.

harmonic signals are measured either as a function of the detection phase (for determination of the IM-FM phase shift) or as a function of the modulation amplitude (for determination of the FM efficiency). The experimental results are compared with the interferometric method described in Section 2.

\section{A. Experimental Measurement of the IM-FM Phase Shift}

To extract the IM-FM phase shift we measure the amplitudes $s_{2, \text { ampl }}$ and $s_{3, \text { ampl }}$ of the second- and third-harmonic signals as functions of detection phase $\Phi_{n}$, as described in Subsection 3.A. Figure 6 shows the experimental measurements obtained for two modulation frequencies $(f=2.5 \mathrm{kHz}$ and $f=15.8$ $\mathrm{kHz}$ ). The difference in the positions of the minima of the two curves corresponds to phase shift $\Psi$. The curves given by our theoretical model are also represented and match the experimental points.

The IM-FM phase shift was measured by this technique over a wide range of modulation frequencies. The results are shown in Fig. 7 and are compared with the interferometric method described above. The results of the two techniques are in excellent agreement in the frequency range considered. The phase shift decreases monotonically with the modulation frequency and goes approximately to zero at low frequencies. This behavior is due to two different effects that contribute to the laser FM. ${ }^{27,28}$ The first one is a thermal effect (the emission frequency varies with the laser temperature owing to the expansion of the cavity), for which the efficiency decreases with increasing modulation frequency. The second is due to the carrier density in the semiconductor laser (the emission frequency varies with the carrier density owing to a change in the refractive index). This effect is fast and is present up to high frequencies, but it is smaller in magnitude and $180^{\circ}$ out of phase compared with the thermal effect. The two effects add to produce the laser FM and the

Table 1. Comparison of the Line Parameters of the $R 16 \mathrm{CO}_{2}$ Line Obtained Experimentally and According to the HITRAN Database

\begin{tabular}{|c|c|c|c|c|}
\hline Line Parameter & Symbol & Experimental Value & $\begin{array}{l}\text { HITRAN } \\
\text { Value }\end{array}$ & Unit \\
\hline Central frequency & $v_{\text {line }}$ & 4989.971 & 4989.973 & $\mathrm{~cm}^{-1}$ \\
\hline Linewidth & $\Delta v_{\text {line }}$ & 0.07324 & 0.07327 & $\mathrm{~cm}^{-1}$ \\
\hline Line intensity & $S$ & $1.341 \times 10^{-21}$ & $1.328 \times 10^{-21}$ & $\mathrm{~cm}^{-1} /\left(\mathrm{mol} \mathrm{cm}{ }^{-2}\right)$ \\
\hline
\end{tabular}


(a)

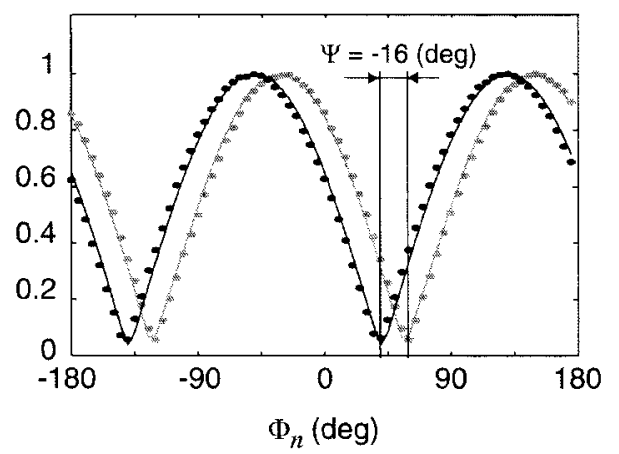

(b)

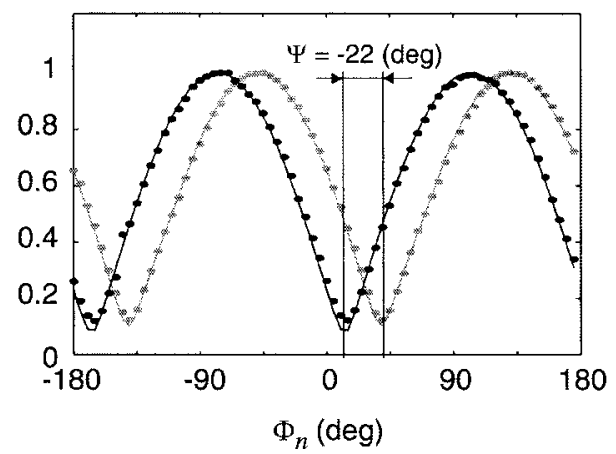

Fig. 6. Evolution of the amplitude of the WMS signal at $2 f$ (lighter curves and circles) and $3 f$ (darker curves and circles) as a function of the detection phase for (a) $f=2.5 \mathrm{kHz}$ and (b) $f=15.8 \mathrm{kHz}$. In both cases, $m=1$. Circles represent experimental measurements, and curves are the results of our theoretical model.

IM-FM phase shift. Consequently the phase shift tends to zero at low frequency and then decreases monotonically as a function of the frequency because of time averaging of the thermal effect.

\section{B. Experimental Measurement of FM Efficiency}

The maximum $s_{2, \max }$ of the second-harmonic signal is measured as a function of laser modulation amplitude $\Delta i$ and for detection phase $\Phi_{2 \text { max }}$ that maximizes the $2 f$ signal. Figure 8 shows the experimental measurements obtained for different modulation frequencies $(f=1 \mathrm{kHz}$ and $f=11 \mathrm{kHz})$. Modulation amplitude $\Delta i$ is linked to modulation in-

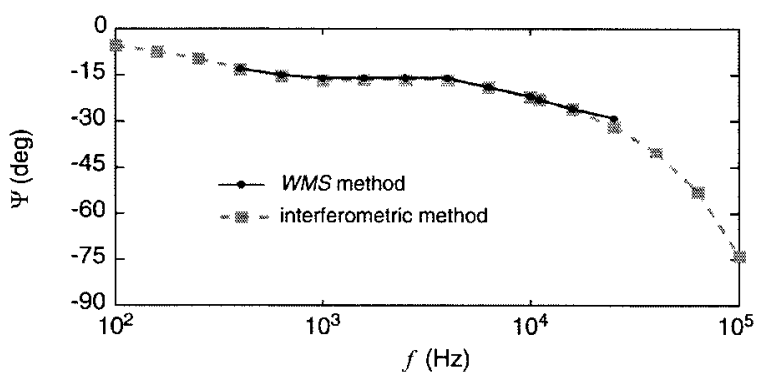

Fig. 7. IM-FM phase shift as a function of modulation frequency: comparison of WMS and interferometric methods. dex $m$ through a fit of the experimental points by Eq. (14), which describes curve $s_{2 \text { max }}(m)$. FM efficiency $\Delta \nu / \Delta i$ is finally determined from absorption linewidth $\Delta v_{\text {line }}$ through Eq. (16). The value of the linewidth was measured experimentally and was confirmed by the HITRAN database. ${ }^{26}$

The FM parameter was measured by this technique for different modulation frequencies. The results are shown in Fig. 9 and are compared with those of the interferometric method described above. The results of the two methods are in excellent agreement in the frequency range considered.

\section{Discussion and Conclusions}

Experimental methods based on wavelengthmodulation spectroscopy have been demonstrated for the characterization of the combined IM-FM of a laser diode. These techniques are based on the analysis of the harmonic signals produced when a modulated laser field interacts with an absorption line of a gas near atmospheric pressure. The theoretical description of these methods is based on a model of WMS that we have developed in the general case of combined IM-FM with an arbitrary phase shift. This theoretical model is valid in the Lorentzian line-shape limit, but the experimental methods (a)

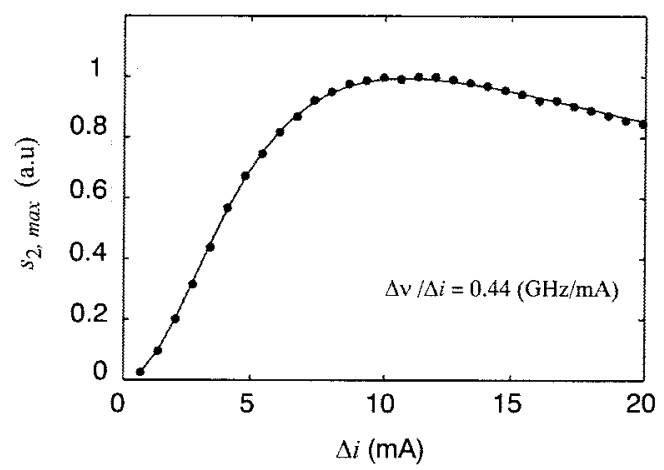

(b)

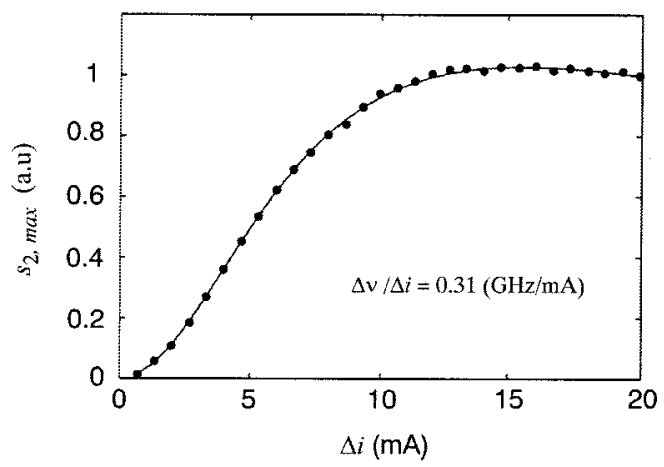

Fig. 8. Evolution of the maximum of the $2 f$ signal as a function of the modulation amplitude for (a) $f=1 \mathrm{kHz}$ and (b) $f=11 \mathrm{kHz}$. Circles, experimental measurements; curves, results of a fit. 


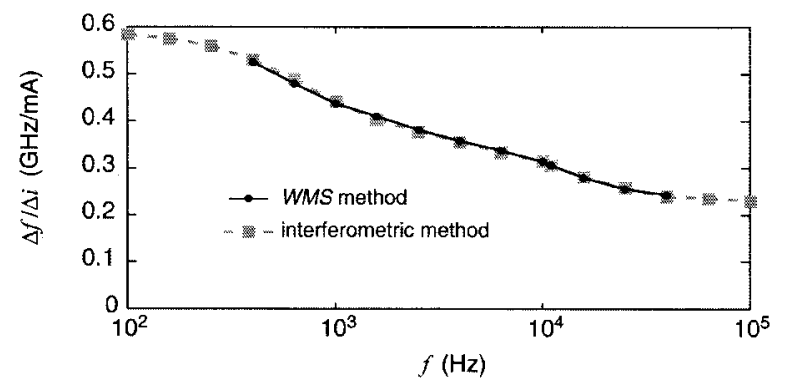

Fig. 9. Parameter $\Delta v / \Delta i$ as a function of modulation frequency: comparison of WMS and interferometric methods.

reported here have in fact a much wider application range. For example, the measurement of the laser IM-FM phase shift with the technique presented in Subsection 3.A [Eq. (11)] remains valid for a Dopplerbroadened absorption line (Gaussian line-shape function) and therefore also for a generalized Voigt profile. The measurement of the FM amplitude with the method reported in Subsection 3.B, however, cannot be applied for a Gaussian line shape, as the maximum of the $2 f$ signal is no longer described by Eq. (14) for a Gaussian absorption line. However, the proposed technique remains applicable for a Voigt profile, when reduced broadening parameter $Y$ (=Lorentz HWHM/Doppler HWHM) is larger than 2.

Two different measurement techniques have been proposed for determination of the FM amplitude on one hand and of the IM-FM phase shift on the other hand. These techniques have been demonstrated experimentally for characterization of the modulation parameters of a 2- $\mu$ m distributed-feedback laser with a $\mathrm{CO}_{2}$ absorption line at atmospheric pressure. The results were compared with those obtained by a traditional interferometric method, and the values of the two techniques are in excellent agreement in the frequency range $(400 \mathrm{~Hz}-30 \mathrm{kHz})$ considered. This comparison proves the validity of the reported measurement techniques.

The methods presented in this paper complement other existing techniques in laser metrology. They can be used to characterize the modulation properties of laser diodes emitting in different spectral ranges, including the optical telecommunications wavelengths, by use of a suitable absorbing gas. For example, HF can be used in the 1260-1350-nm range; $\mathrm{C}_{2} \mathrm{H}_{2}, \mathrm{HCN}$, and $\mathrm{CO}$ to cover the third windows of optical fibers (1510-1600 nm); and $\mathrm{CH}_{4}$ near 1650 nm. Furthermore, this technique can also be implemented by use of the resonance of an optical etalon (a Fabry-Perot cavity) instead of a gas absorption line, which extends its range of application to any wavelength in the infrared. In our setup, this technique was limited to modulation frequencies lower than approximately $30 \mathrm{kHz}$ because of the bandwidth limit of our lock-in amplifier. However, the use of a rf lock-in or a rf mixer enables this range to be extended to much higher frequencies. The modulation frequency should, however, stay much smaller than the absorption linewidth, so the WMS description is valid. When the modulation frequency is of the same order of magnitude or higher than the absorption linewidth, the technique is referred as frequency modulation spectroscopy, and another theoretical description must be considered.2,23

Another important point of the experimental method reported in this paper concerns knowledge of the absorption linewidth. This parameter should be known precisely for determination of the FM efficiency, as these two factors are directly proportional [see Eq. (16)]. However, the absorption linewidth is not relevant for determination of the IM-FM phase shift and thus causes no inaccuracy; the measurement can be made even if the linewidth is unknown. Finally, another advantage of the methods reported here compared with traditional techniques concerns the required bandwidth of the detector that is used to measure the signals. The interferometric technique reviewed in Section 2 requires a fast detector, especially when high precision of the parameters $\Delta \nu / \Delta i$ and $\Psi$ is necessary. A large number of interference fringes should be detected to obtain good precision on the parameters. Typically, 20-40 fringes per modulation half-period are necessary. This implies the use of a photodetector with a bandwidth approximately 100 times higher than the modulation frequency. The methods reported that are based on WMS require a much lower bandwidth, as it is sufficient to detect the second and third harmonics of the modulation frequency.

\section{References}

1. J. Reid and D. Labrie, "Second-harmonic detection with tunable diode lasers-comparison of experiment and theory," Appl. Phys. B 26, 203-210 (1981).

2. J. M. Supplee, E. A. Whittaker, and W. Lenth, "Theoretical description of frequency modulation and wavelength modulation spectroscopy," Appl. Opt. 33, 6294-6302 (1994).

3. D. T. Cassidy and L. J. Bonnell, "Trace gas detection with short-external-cavity InGaAsP diode laser transmitter modules operating at $1.58 \mu \mathrm{m}$," Appl. Opt. 27, 2688-2693 (1988).

4. A. Lucchesini, I. Longo, C. Gabbanini, S. Gozzini, and L. Moi, "Diode laser spectroscopy of methane overtone transitions," Appl. Opt. 32, 5211-5216 (1993).

5. X. Zhu and D. T. Cassidy, "Modulation spectroscopy with a semiconductor diode laser by injection-current modulation," J. Opt. Soc. Am. B 14, 1945-1950 (1997).

6. D. T. Cassidy and J. Reid, "Atmospheric pressure monitoring of trace gases using tunable diode lasers," Appl. Opt. 21, 11851190 (1982).

7. D. S. Bomse, A. C. Stanton, and J. A. Silver, "Frequency modulation and wavelength modulation spectroscopies: comparison of experimental methods using a lead-salt diode laser," Appl. Opt. 31, 718-731 (1992).

8. J. Reid, B. K. Garside, M. El-Sherbiny, and E. A. Ballik, "High sensitivity point monitoring of atmospheric gases employing tunable diode lasers," Appl. Opt. 17, 1806-1810 (1978).

9. M. Loewenstein, "Diode laser harmonic spectroscopy applied to in situ measurements of atmospheric trace molecules," J. Quant. Spectrosc. Radiat. Transfer 40, 249-256 (1988).

10. F. S. Pavone and M. Inguscio, "Frequency- and wavelengthmodulation spectroscopies: comparison of experimental methods using an AlGaAs diode laser," Appl. Phys. B 56, 118122 (1993).

11. K. Namjou, S. Cai, E. A. Whittaker, J. Faist, C. Gmachl, F. 
Capasso, D. L. Sivco, and A. Y. Cho, "Sensitive absorption spectroscopy with a room-temperature distributed-feedback quantum-cascade laser," Opt. Lett. 23, 219-221 (1998).

12. R. M. Williams, J. F. Kelly, S. W. Sharpe, J. S. Hartman, C. Gmachl, F. Capasso, D. L. Sivco, J. N. Baillargeon, and A. Y. Cho, "Spectral and modulation performance of quantum cascade lasers with application to remote sensing," in Application of Tunable Diode and Other Infrared Sources for Atmospheric Studies and Industrial Processing Monitoring II, A. Fried, ed., Proc. SPIE 3758, 11-22 (1999).

13. C. R. Webster, G. J. Flesch, D. C. Scott, J. E. Swanson, R. D. May, W. S. Woodward, C. Gmachl, F. Capasso, D. L. Sivco, J. N. Baillargeon, A. L. Hutchinson, and A. Y. Cho, "Quantumcascade laser measurements of stratospheric methane and nitrous oxide," Appl. Opt. 40, 321-326 (2001).

14. M. Gabrysch, C. Corsi, F. S. Pavone, and M. Inguscio, "Simultaneous detection of $\mathrm{CO}$ and $\mathrm{CO}_{2}$ using a semiconductor diode laser at $1.578 \mu \mathrm{m}$," Appl. Phys. B 65, 75-79 (1997).

15. A. Lucchesini, M. De Rosa, D. Pellicia, A. Ciucci, C. Gabbanini, and S. Gozzini, "Diode laser spectroscopy of overtone bands of acetylene," Appl. Phys. B 63, 277-282 (1996).

16. U. Gustafsson, G. Somesfalean, J. Alnis, and S. Svanberg, "Frequency-modulation spectroscopy with blue diode lasers," Appl. Opt. 39, 3774-3780 (2000).

17. G. Jacobsen, H. Olesen, and F. Birkedahl, "Current/frequencymodulation characteristics for directly optical frequencymodulated injection lasers at $830 \mathrm{~nm}$ and $1.3 \mu \mathrm{m}$," Electron. Lett. 18, 874-876 (1982).

18. H. Olesen and G. Jacobsen, "A theoretical and experimental analysis of modulated laser fields and power spectra," IEEE J. Quantum Electron. 18, 2069-2080 (1982).

19. M. Imai and K. Kawakita, "Measurement of direct frequency modulation characteristics of laser diodes by Michelson interferometry," Appl. Opt. 29, 348-353 (1990).

20. H. Tsuchida, T. Tako, and M. Ohtsu, "A novel technique for measuring the frequency deviation of semiconductor lasers under direct modulation," Jpn. J. Appl. Phys. 22, L19-L21 (1983).

21. H. Olesen and G. Jacobsen, "Phase delay between intensity and frequency modulation of a semiconductor laser (including a new measurement method)," in Proceedings of the 8th European Conference on Optical Communication (n.p., 1982), pp. 291-295.

22. S. Schilt, L. Thévenaz, and P. Robert, "Wavelength modulation spectroscopy: combined frequency and intensity laser modulation," Appl. Opt. 42, 6728-6738 (2003).

23. S. Schilt, "Mesure de traces de gaz à l'aide de lasers à semiconducteur," Ph.D. dissertation (Swiss Federal Institute of Technology, Lausanne, 2002).

24. P. Kluczynski, J. Gustafsson, A. Lindberg, and O. Axner, "Wavelength modulation absorption spectrometry-an extensive scrutiny of the generation of signals," Spectrochim. Acta B 56, 1277-1354 (2001).

25. R. Arndt, "Analytical line shapes for Lorentzian signals broadened by modulation," J. Appl. Phys. 36, 2522-2524 (1965).

26. L. S. Rothman, C. P. Rinsland, A. Goldman, S. T. Massie, D. P. Edwards, J.-M. Flaud, A. Perrin, C. Camy-Peyret, V. Dana, J. Y. Mandin, J. Schroeder, A. McCann, R. R. Gamache, R. B. Wattson, K. Yoshino, K. V. Chance, K. W. Jucks, L. R. Brown, V. Nemtchinov, and P. Varanasi, "The HITRAN molecular spectroscopic database and HAWKS (HITRAN atmospheric workstation): 1996 edition,” J. Quant. Spectrosc. Radiat. Transfer 60, 665-710 (1998).

27. F. Wittgrefe, M. D. Hoogerland, and J. P. Woerdman, "Semiconductor lasers for spectroscopy,” Meas. Sci. Technol. 2, 304311 (1991).

28. S. Saito, O. Nilsson, and Y. Yamamoto, "Coherent FSK transmitter using a negative feedback stabilized semiconductor laser," Electron. Lett. 20, 703-704 (1985). 